\title{
One Continuous Auditing Practice in China: Data-oriented Online Auditing(DOOA)
}

\author{
Wei Chen, Jin-cheng Zhang, and Yu-quan Jiang \\ Nanjing Audit University, Nanjing, Jiangsu 210029, China \\ chenweich@nau.edu.cn \\ WWW home page: http://info.nau.edu.cn
}

\begin{abstract}
Application of information technologies (IT) in the field of audit is worth studying. Continuous auditing (CA) is an active research domain in computer-assisted audit field. In this paper, the concept of continuous auditing is analyzed firstly. Then, based on analysis on research literatures of continuous auditing, technique realization methods are classified into embedded mode and separate mode. According to the condition of implementing online auditing in China, data-oriented online auditing (DOOA) used in China is also one of separate mode of continuous auditing. And the principle of DOOA is analyzed. Furthermore, the advantages and disadvantages of DOOA are also discussed. Finally, advices to implement DOOA in China are given, and the future research topics related to continuous auditing are also discussed.
\end{abstract}

\section{Introduction}

Auditing is a part of control process in organizations, which can be used to examine and observe the reliability of the accounts and that they give a true and fair view of the auditee's result of operations and financial position. Therefore, it is very important to a country. As computer technology has advanced, government organizations have become increasingly dependent on computerized information systems to carry out their business operations and service delivery and to process, maintain and report essential information.

In order to finish audit work in informationization environment, computerassisted audit technologies [1-3] must be used. With the development of information technologies (IT), IT applications in audit area are also changing. The development of IT will make computer-assisted audit become continuous, dynamic and real-time.

Please use the following format when citing this chapter:

Chen, W., Thang, J.-C., Jiang, Y.-Q., 2007, in IFIP International Federation for Information Processing, Volume 252, Integration and Innovation Orient to E-Society Volume 2, eds. Wang, W., (Boston: Springer), pp. 521-528. 
Koskivaara[4] has developed five stages of information technology utilization in auditing. At stage one, standard software applications are used. At stage two, some databases, email, and graphics are also adapted. At stage three, several different external and internal databases, audit software applications, and company models are in use. At stage four, expert systems, decision support systems, and special audit software for continuous auditing are utilized. At the fifth stage, the software applications are based on advanced methods like ANNs (artificial neural networks), fuzzy logic, and genetic algorithms. These advanced methods could produce information for continuous monitoring and controlling. Therefore, continuous auditing becomes an important research trend in computer-assisted audit field. Although the thoughts of continuous auditing have been proposed for many years, the developments of IT recently make continuous auditing feasible. Continuous auditing has attracted a lot of attention from researchers, auditors and software developers over the past three decades. Continuous auditing research center was also founded in Rutgers, the state university of New Jersey, and the meeting on continuous auditing is held every year. As a consequence, Continuous auditing researches develop quickly.

In China, in order to meet the requirements of computer-assisted audit, some researches on continuous auditing were also done. In this paper, the researches on continuous auditing are analyzed firstly. Then, the realization methods of continuous auditing are summarized and classed. Finally, DOOA, one realization methods of continuous auditing in China are studies, the advantages and disadvantages of DOOA are also discussed, and advices to implement DOOA are given.

\section{Concept and Category of Continuous Auditing}

Continuous auditing was defined in some literatures. CICA (the Canadian Institute of Chartered Accountants) and AICPA (the American Institute of Certified Public Accountants) defined continuous auditing as "a methodology that enables the auditor to provide assurance on a subject matter simultaneously with, or very shortly after, the occurrence of events underlying the subject matter [5]." Alexander[6] thought that continuous auditing was defined here as a type of auditing that produces audit results simultaneously with, or a short period of time after, the occurrence of relevant events. According to this define, Alexander thought continuous auditing would be more accurate to call this type of auditing instant rather than continuous. Furthermore, Alexander also thought that the only known way to make continuous auditing feasible is to implement continuous auditing on an online computer system. So, continuous auditing can also be called continuous online auditing (COA). One realization method of off-site audit in informationization environment is researched [7]. This style of off-site audit is also one mode of continuous auditing.

In a word, researches of continuous auditing mainly concentrate on realization methods, theory analysis and key technologies. And most researches are about realization methods. The realization principle of continuous auditing is different in different periods and different technology conditions. According to [8], continuous auditing methodology can be categorized into two major streams: stand-alone 
systems that continuously monitor auditee systems, extract data from those systems, compare data patterns with standards, trigger alarms/report exceptions, and ultimately achieve the goal of auditing; and sub-systems or modules that must be embedded into auditee systems.

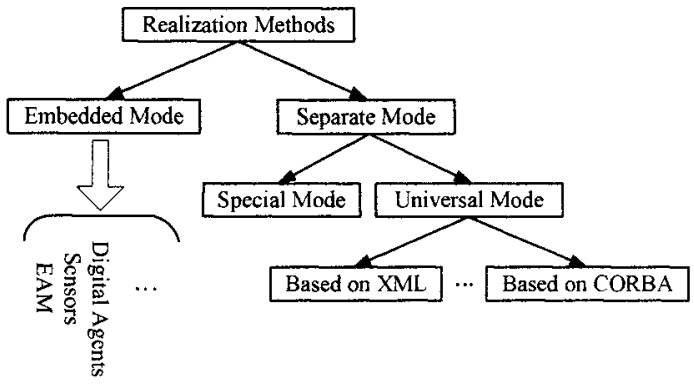

Fig. 1. The sorts of realization methods on continuous auditing

Based on analysis on research literatures of continuous auditing, realization methods of continuous auditing are classed as fig. 1 in this paper. That is to say, realization methods of continuous auditing can be classed into two major kinds: embedded mode (such as $[9,10]$ ) and separate mode (such as $[11,12]$ ). Embedded mode is realized using EAM, sensors, digital agents, and so on. Separate mode is mainstream. According to the flexibility of continuous auditing system, realization methods of separate mode continuous auditing also can be classed into special mode and universal mode. Special mode is a realization method designed for one continuous auditing system, and cannot be used for another continuous auditing system. Universal mode is a realization method that is universal and can be used for many continuous auditing systems. The methods used in universal mode include methods based on XML, methods based on CORBA, and so on.

\section{Data-oriented Online Auditing in China}

The National Audit Office of China (CNAO) is dedicated in researching, realizing, popularizing and applying online auditing techniques and attempts to change the ways of audit work in recent years in China.

In China, in order to adapt the requirement of computer-assisted audit, the National Audit Office of China (CNAO) has implemented the first period of "Jinshen Project" successfully. The "Jin-shen Project" is also means the "Golden Auditing Project", which is one of the 12 key IT business systems defined by the State Council under China's e-Government framework.

In a short, the "Jin-shen Project" is Chinese audit informationization. The main aim of the "Jin-shen Project" is to discovery the new audit mode; carry out a new online auditing model; and achieve three "transformations", which are to move from ex post audit to a combination of ex post audit and continuous audit; from static audit 
to a combination of static and dynamic audit; and from on-the-spot audit to a combination of on-the-spot and remote audit.

Through the first period of "Jin-shen Project", the infrastructures of audit departments, such as software and hardware, have been constructed; some audit software have been developed; and auditors have been trained to familiar with database, network, and so on.

Furthermore, in order to prepare for implementing the second period of "Jin-shen Project", the National Audit Office of China (CNAO) also has finished two research projects of the National High-Tech Research and Development Plan of China "data acquisition and process of IT audit". Through these two projects, online auditing technologies that adapt to Chinese condition are researched.

In China, online auditing is using network computer techniques to collect the auditee' data and transmit data to auditor' department. Then, auditors can process the auditee' data in their own department. And it is data-oriented. So, it is called dataoriented online auditing (DOOA). The principle of DOOA is shown in Fig.2. From Fig.2, we can see that the realization method of online auditing researched in China is also one mode of continuous auditing. It is one of separate mode of continuous auditing.

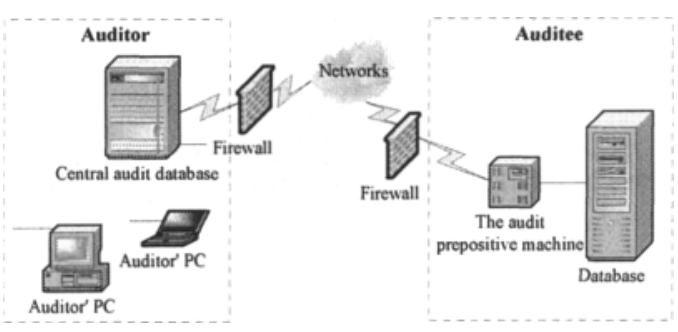

Fig. 2. The principle of continuous audit in China

Fig. 2 shows that DOOA has 4 steps [13]:

(1) Audit data acquisition

In order to effectively implement the audit supervision in online auditing, it is necessary to collect the auditee' data timely. Auditors should not depend on monthly backup data, or yearly backup data of auditee. So, a PC server can be set at auditees, which is called audit prepositive machines, to collect the auditee' data according to the need of auditors. Through setting the audit prepositive machine appropriately, auditors can collect the auditee' data that auditors need and store in the audit prepositive machine.

(2) Audit data transmission

After finishing data acquisition, the audit prepositive machine will transmit the auditee' data to auditors' PC server through networks according to auditors' command.

(3) Audit data storage 
Data collected from auditee increase massively under the condition of DOOA. It makes a good basis for mastering auditees' situations and also provides convenience to support other audit projects. To improve the efficiency of audit supervision, online storage and near-line storage are adopted in auditors' data storage. And data storage methods used in DOOA are researched in [14].

(4) Audit data processing

Audit data processing is an important step in DOOA. Through data processing, doubtful data can be found from the auditee' data.

Nowadays, in China, auditors usually use audit software, through using analysis methods such as browse, selection, comparison and check, sampling, statistics and gap, etc, to analyze data. And Audit Office (AO) is one of important audit software, which is developed by IT Center of CNAO. Audit Office is an integrated software, which integrates many data acquisition and analysis methods of other audit software.

However, the volume and complexity of accounting transactions in major organizations have increased dramatically. To audit such organizations, auditors frequently must deal with voluminous data with rather complicated data structure. Consequently, auditors can use technologies such as OLAP (On-Line Analytical Processing) to analyze the auditee' data. Rather, additional tools such as data mining techniques that can automatically extract information from a large amount of data might be very useful.

\section{Advantages and Disadvantages of Implementing DOOA}

In this section, we will discuss advantages and disadvantages of DOOA. According to analysis on DOOA above, we can see that advantages of implementing DOOA are as follows.

(1) Implementing DOOA can eliminate seven types of audit wastes.

Usually, there are seven types of audit wastes that can occur in the current audit domain, which are over auditing, waiting, time delays, the audit process, work-inprocess, review process, errors and mistakes. DOOA is one mode of CA. So, these seven types of audit wastes can be eliminated through implementing DOOA.

(2) Implementing DOOA can reduce the audit cost.

If DOOA is implemented, the number of auditors will be reduced, so, the costs that spend on auditors will also be reduced. For some audit projects that are not in the same place with auditors, the outlay such as the cost of go on errands, the cost of hotel, and so on, will be reduced through implementing DOOA.

(3) Implementing DOOA can save the audit time and improve the efficiency of audit.

Because the condition of auditee is usually complicated, all problems cannot be found through one auditing in traditional audit mode. Through implementing DOOA, the auditee's data are collected and auditors can use the advanced audit methods, such as data mining, to analyze the auditee's data carefully. Therefore, the problems can be found roundly.

However, implementing DOOA also has some disadvantages. And disadvantages of DOOA are as follows. 
(1) The cost of implementing DOOA is high.

The cost of implementing DOOA can be categorized into two kinds: one is oneoff cost, and another is regular cost. One-off cost is investment of developing and implementing initially online audit system. Regular cost is investment of using and maintaining online auditing system in all life of online auditing system. Through the realization principle of DOOA, we can see that the cost of implementing DOOA is high.

(2) The techniques need of implementing DOOA is high.

DOOA is realized using information technologies in China. In order to ensure the progress of DOOA, auditors should understand each parts of DOOA, such as data acquisition, data transmission, data storage, and data process, which requires that auditors should familiar with software, hardware, network and databases.

(3) The audit risk of implementing DOOA is high.

The objects of auditing are electronic data that are collected from the auditee's information systems in DOOA. However, these electronic data needed to be ensured by internal control. If there are not efficient internal control systems to ensure the facticity of these electronic data, auditors will get wrong results from illusive electronic data, which will cause great audit risk.

Furthermore, DOOA system is also a complex system. Sometimes, some disasters will happen, which can cause DOOA system pause. The pauses of DOOA system will influent auditing work. So, the audit risk of implementing DOOA is high.

\section{Advices on Implementing DOOA in China}

According to the characters of DOOA implementing in China, some problems that should be paid attention are as follows:

(1) When choosing the auditees to implement DOOA, some aspects should be considered: (1) For state audit, the auditees to implement DOOA should be important departments that are audited frequently, which can make DOOA more valuable. For internal audit in some large enterprises, DOOA also can be implemented if circumstance is feasible; (2) In order to reduce audit risk, the internal control system of auditee should be perfect to ensure that actual data can be collected by DOOA system. (3) The degree of informationization in auditee should be higher, and finance software should be normative, which are basal condition to implement DOOA. (4) Under condition of safe network transmission, the cost of implementing DOOA between Auditor and auditee should be cheaper.

(2) Universal and portable DOOA software should be designed when DOOA system is developed. So, the audit implementing cost can be reduced. For example, it is important to research how to design universal audit data acquisition interface, such as interface based on XBRL, or how to design reconfigurable audit data acquisition system, which can make DOOA system universal.

(3) According to the condition of auditee, other proper methods also can be used when realization method presented in this paper is not feasible. For example, EAMs (Embedded Audit Modules) mode can be used for some ERP (Enterprise Resource Planning) systems in order to implement online auditing. 


\section{Conclusions}

Continuous auditing is one new audit mode in informationization environment. Developments in information technology enabled continuous auditing feasible. In a words, implementing continuous auditing can improve the efficiency of auditing, reduce the audit cost, improve the quality of auditing, extend the scope of auditing, and make auditing work standardization. With developments in continuous auditing, traditional audit mode will be impacted, although continuous auditing cannot substitute traditional audit mode completely.

However, there are many problems on continuous auditing that need be studied deeply. For example, some researches are as follows:

(1) Research on key technologies of continuous auditing.

In order to implement continuous auditing, more technologies, such as data processing methods that are more efficient and adaptive to continuous auditing, should be researched.

(2) Research on how to make realization methods of continuous auditing universal and portable.

For example, for separate mode of continuous auditing, it is important to research how to design universal audit data acquisition interface, such as interface based on $\mathrm{XBRL}$, or how to design reconfigurable audit data acquisition system, which can make continuous auditing system universal.

(3) Research on realization methods of continuous auditing for special trade.

For example, current versions of ERP systems neither include EAMs nor provide any real continuous auditing capability [15]. So, it is important to research realization methods of continuous auditing on ERP system.

Now, supported by the National High-Tech Research and Development Plan of China and China Postdoctoral Science Foundation, we are doing some researches on DOOA, and hope make some contributions to develop continuous auditing.

\section{Acknowledgement}

This work is supported by China Postdoctoral Science Foundation (No. 20060390281); the Natural Science Research Programs of college of Jiangsu Province (No. 05KJB520054); Jiangsu Planned Projects for Postdoctoral Research Funds (No. 0502023C); the National High-Tech Research and Development Plan of China (No. 2005AA1Z2140).

\section{References}

1. M. L. Linda. "Audit Technology and the Use of Computer Assisted Audit Techniques." Journal of Information Systems 4 (2), 60-68 (1990).

2. L. B. Robert, E. D. "Harold. Computer-assisted Audit Tools and Techniques: Analysis and Perspectives". Managerial Auditing Journal 18 (9), 725-731 (2003). 
3. INTOSAI audit committee. Principles of Computer Assisted Audit Techniques - Student Notes, 2004, (unpublished).

4. E. Koskivaara. Artificial Neural Networks for Analytical Review in Auditing. Finland: Turku School of Economics and Business Admistration, (2004).

5. CICA/AICPA. Continuous Auditing Research Report. The Canadian Institute of Chartered Accountants, Toronto, Ontario, (1999).

6. K. Alexander, F. S. Ephraim, and A. V. Miklos. "Continuous Online Auditing: a Program of Research". Journal of Information Systems 13(2), 87-103 (1999).

7. Wang Huijin, Chen wei. Study on the realization method of off-site audit. Audit and Economy Research 20(3), 36-39 (2005).

8. H. Du, S. Roohani. A Framework for Independent Continuous Auditing of Financial Statements. In: American Accounting Association 2006 Annual Meeting. Washington, (2006).

9. S. M. Groomer, U. S. Murthy. "Continuous Auditing of Database Applications: An Embedded Audit Module Approach". Journal of Information Systems 3(2), 53-69 (1989).

10. R. S. Debreceny, G. L. Gray, J. Ng, et al. "Embedded Audit Modules in Enterprise Resource Planning Systems: Implementation and Functionality". Journal of Information Systems, 19(2), 7-27 (2005).

11. Z. Rezaee, A. Sharbatoghlie, and R. Elam, etc. "Continuous Auditing: Building Automated Auditing Capability". Auditing: A Journal of Practice and Theory 21(1), 147-163 (2002).

12. U. S. Murthy, S. M. Groomer." A Continuous Auditing Web Services Model for XMLbased Accounting Systems". International Journal of Accounting Information System 5(2), 139-163 (2004).

13. Chen wei, Wang hao, Zhu Wenming. Study on Data-oriented IT Audit Used in China. In: Proceedings of the 11 th Joint International Computer Conference. Singapore: World Scientific Publishing, pp. $666-669$ (2005).

14. Wu Haiping, Yu Hongliang, Zheng Weimin, etc. "A Massive Data Storage and Management Strategy for Online Computer-assisted Audit System". Chinese Journal of Computer 29(4), 618-624 (2006).

15. M. G. Alles, A. Kogan, M. A. Vasarhelyi. "Feasibility and Economics of Continuous Assurance". Auditing: A Journal of Theory and Practice 21 (1), 125-138 (2002). 